\title{
A Drug Release Mechanism Controlled by Hydrophobic/ Hydrophilic Balance of the Matrix. Theoretical and Experimental Perspectives
}

\author{
LOREDANA HIMINIUC ${ }^{1}$, MARICEL AGOP ${ }^{2,3}$, VLAD GHIZDOVAT ${ }^{4}$, \\ MARIA-ALEXANDRA PAUN ${ }^{5}$, VLADIMIR-ALEXANDRU PAUN ${ }^{6}$, \\ CONSTANTIN BACIU ${ }^{7}$, VIOREL-PUIU PAUN ${ }^{8,3^{*}}$, BOGDAN TOMA ${ }^{1}$ \\ ${ }^{1}$ "Grigore T. Popa" University of Medicine and Pharmacy Iasi, Department of Obstetrics and Gynecology, 16 Universitatii \\ Str., 700115, Iasi, Romania \\ ${ }^{2}$ Gheorghe Asachi” Technical University, Physics Department, 59A Prof. dr. docent Dimitrie Mangeron Blvd., 700050, Iasi, \\ Romania \\ ${ }^{3}$ Academy of Romanian Scientists, 54 Splaiul Independentei, 050094, Bucharest, Romania \\ 4"Grigore T. Popa" University of Medicine and Pharmacy, Faculty of Medicine, Biophysics and Medical Physics Department, \\ 16 University Str., 700115, Iasi, Romania \\ ${ }^{5}$ School of Engineering, Swiss Federal Institute of Technology (EPFL), Route Cantonale, 1015 Lausanne, Switzerland \\ ${ }^{6}$ Five Rescue Research Laboratory, 35 Quai d'Anjou, 75004, Paris, France \\ ${ }^{7}$ "Gheorghe Asachi” Technical University, Faculty of Science and Material Engineering, 59A, Prof. dr. docent Dimitrie \\ Mangeron Blvd., 700050, Iasi, Romania \\ ${ }^{8}$ Physics Department, Faculty of Applied Sciences, University Politehnica of Bucharest, 313 Splaiul Independentei, 060042, \\ Bucharest, Romania
}

\begin{abstract}
Controlled drug release is a promising pathway of biomedicine, meant to suppress side effects with the aim of increasing patient's comfort. A route to achieve this goal represents the encapsulation of drugs into matrixes, capable to develop physical forces, which further can control the drugs release. To this purpose, mathematical modeling is an important tool, which offers the possibility to understand the drug release mechanisms and to further design new performant systems. In this paper, a theoretical model for drug release from an amphiphilic matrix is presented. This is achieved using a conservation multifractal law of probability density followed by validation of the model. Moreover, because nonsteroidal anti-inflammatory drugs (NSAIDs), such as diclofenac, are widely used in endometriosis as painkillers for dysmenorrhea management or Asherman syndrome for reducing the endometrial inflammation, some implications of our model for drug delivery systems applied in the field of gynecology have been discussed.
\end{abstract}

Keywords: controlled drug release, drug delivery systems, amphiphilic matrix, nonsteroidal antiinflammatory drugs, diclofenac

\section{Introduction}

Chitosan is a biopolymer used in a large area of applications due to its beneficial properties such as biocompatibility, biodegradability and antimicrobial activity [1-5]. Among these applications, its use as a matrix for drug delivery holds the promise to overcome the side effects of the systemic administration, i.e. nausea, vomiting, diarrhea, or even hepatotoxicity [6-12]. This is due to the polycationic nature of chitosan which favors a strong anchoring of the drug molecules by Coulomb forces, and also by formation of H-bonds with the hydroxyl groups [7-10]. The development of such interfacial forces competes with the hydrogen bonds developed between the drug molecules and water solvent, assuring a slow release. The main drawback of the use of chitosan for drug delivery systems is its low solubility at

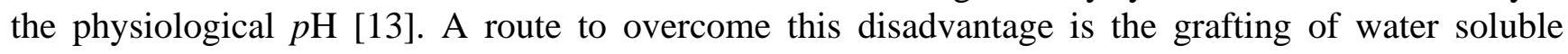
poly(ethylene glycol) (PEG) chains on the chitosan backbones, by obtaining PEGylated chitosan [9].

*email:viorel.paun@physics.pub.ro,viorel_paun2006@yahoo.com 
Replacing chitosan with PEGylated chitosan in view of the development of matrixes for controlled drug release, not only suppresses the disadvantage of the chitosan hydrophobicity in neutral or basic $\mathrm{pH}$ media, but also creates an important tool towards the control of the drug release rate by simple control of the hydrophobic/hydrophilic balance $[9,14]$. In this line of thoughts, an amphiphilic matrix based on chitosan was prepared and also its ability to release a model drug in controlled manner was investigated [9]. It was demonstrated that the degree of substitution of the chitosan backbones with PEG chains tune the drug release rate by the dissolution rate. These systems demonstrated lack of any in vivo toxicity, encouraging further investigation of the laws which govern their ability to function as an efficient matrix.

A large study found a statistically significant increased risk of various adverse cardio-vascular effects such as atrial fibrillation, ischemic events and vascular deaths after NSAIDs use but mainly after diclofenac intake. The authors indicated that the effects of various treatments in specific patients can be forecasted, helping in guiding decisions in inflammatory pathologies [15-17]. Another large study showed 50\% rate of side effects among diclofenac initiators compared with $20 \%$ among paracetamol group or 30\% among naproxen initiators compared with non-initiators [18]. However, the therapeutic approach via nanomedicine may reduce these side effect and increase the efficacy of the drugs.

In the present paper a new theoretical model for drug release from an amphiphilic matrix was proposed. The model is based on a set of multifractal conservation laws for probability density. This approach becomes operational if we take into account the fact that the most usual procedure of multifractal presentation is stochasticity. Finally the model will be validated by empirical data.

\section{Materials and methods}

\subsection{Materials}

Diclofenac sodium salt, citral, chitosan (low molecular weight), phosphate buffer saline $(p \mathrm{H}=7.4)$ and ethanol were provided by Sigma Aldrich and used without further purification.

\subsection{Synthesis of the drug delivery systems}

Formulations were prepared by in situ hydrogelation of PEGylated chitosan with citral in the presence of diclofenac sodium salt, following a receipt already published [9]. Shortly, two hydrophilic PEGylated chitosan derivatives with different content of PEG were reacted with hydrophobic citral in the presence of model drug, at $55^{\circ} \mathrm{C}$, under vigorous magnetic stirring. The drug amount was kept constant, while the amounts of PEGylated chitosan and citral varied in order to assure a different ratio of the hydrophilic/hydrophobic components (Scheme 1).

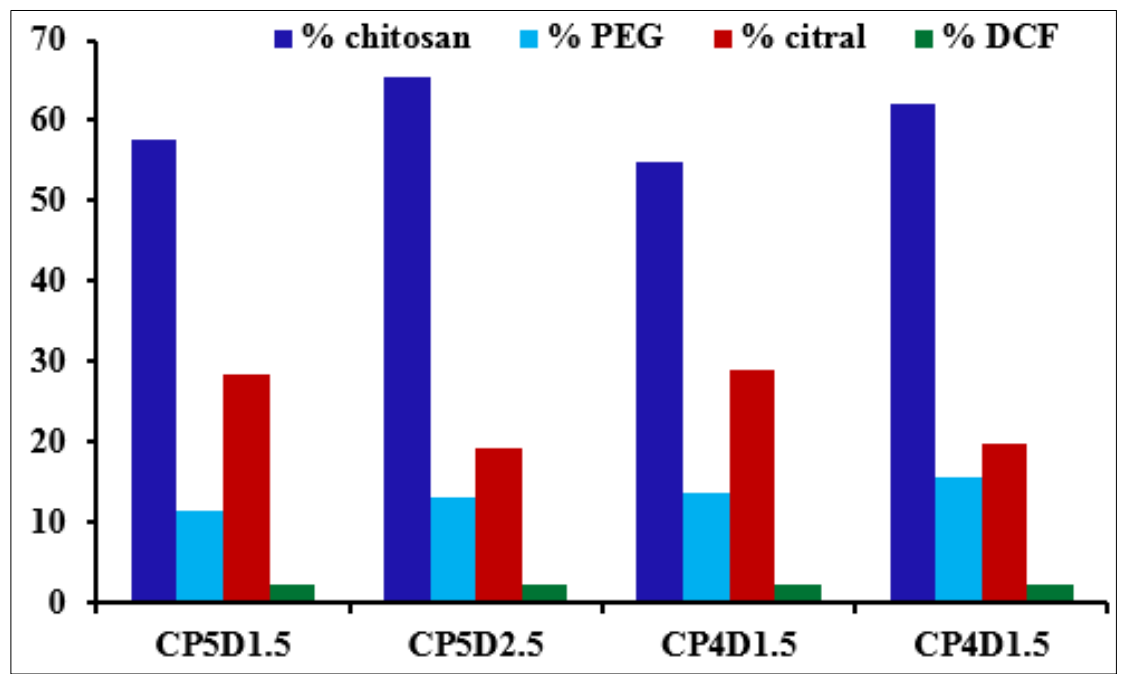

Scheme 1. Graphical representation of the components of the formulations 


\subsection{Methods}

The investigation of the supramolecular architecture of the systems was realized by polarized optical microscopy (POM), using a Leica DM 2500 microscope.

The morphology of the samples was evaluated with a field emission scanning electron microscope (Scanning Electron Microscope SEM EDAX - Quanta 200) at accelerated electron energy of $10 \mathrm{eV}$.

The in vitro release kinetic was monitored by batch experiment performed in phosphate buffer saline (PBS) $(p \mathrm{H}=7.4)$, at the human body temperature $\left(37^{\circ} \mathrm{C}\right)$, following a previously used experimental procedure, which mainly consisted in the determination of the percent of released drug at different moments $[9,17]$. The experiments were done in triplicate and the values were given as the mean value of three independent measurements. The kinetic data was fitted on several mathematic models, as follows:

i.Zero order model: $\mathrm{Q}_{\mathrm{t}}=\mathrm{K}_{0} \cdot \mathrm{t}$, where $\mathrm{Q}_{\mathrm{t}}$ is the amount of drug dissolved in the time $\mathrm{t}$ and $\mathrm{K}_{0}$ is the zero-order release constant.

ii.Higuchi model: $\mathrm{Q}_{\mathrm{t}}=\mathrm{K}_{\mathrm{H}} \cdot \mathrm{t}^{\frac{1}{2}}$, where $\mathrm{Q}_{\mathrm{t}}$ is the amount of drug released in the time $\mathrm{t}$ and $\mathrm{K}_{\mathrm{H}}$ is the Higuchi dissolution constant.

iii.Hixson-Crowell model: $\mathrm{W}_{0}^{1 / 3}-\mathrm{W}_{\mathrm{t}}^{1 / 3}=\mathrm{K} \cdot \mathrm{t}$, where $\mathrm{W}_{0}$ is the initial amount of drug in the formulation, $\mathrm{W}_{\mathrm{t}}$ is the remaining amount of drug in the formulation at time $\mathrm{t}$ and $\mathrm{K}$ is a constant.

iv.Korsmeyer-Peppas model: $\frac{\mathrm{M}_{\mathrm{t}}}{\mathrm{M}_{\infty}}=\mathrm{K} \cdot \mathrm{t}^{\mathrm{n}}$, where $\mathrm{M}_{\mathrm{t}} / \mathrm{M}_{\infty}$ is the fraction of drug released at the time $\mathrm{t}, \mathrm{K}$ is the release rate constant and $\mathrm{n}$ is the release exponent.

v.First order model: $\log Q_{t}=\log Q_{0}+K \cdot t / 2.303$, where $\mathrm{Q}_{\mathrm{t}}$ is the amount of drug released in the time $t, \mathrm{Q}_{0}$ is the initial amount of drug and $\mathrm{K}$ is the first order release constant.

\subsection{Theoretical model}

In the past years a wide range of theoretical models aiming at describing drug release mechanisms have been developed. The first type of models are empirical and semi-empirical models. The most used ones are the zero-order model, Higuchi model, Hixon- Crowell model, Korsmeyer-Peppas model, first order model etc. [18-21]. There are also kinetic models developed on spaces with integer dimensions, i.e. those based on the usual conservation laws for mass, momentum or velocity, or kinetic models developed on spaces with a non-integer dimension, i.e. based on the conservation laws explicitly written through fractional derivatives [22]. Recently, models have been developed based on operational procedures, i.e. on the group invariance of the conservation laws (groups' automorphism and isomorphism, dimensions compactizations, integral invariant functions, embeddings of spaces etc.) [23]. Lastly, a new generation of theoretical models has arisen, based on Scale Relativity, either in the monofractal dynamics as in the case of Nottale [24], or in the multifractal dynamics as is the case for The Multifractal Theory of Motion [25, 26].

In the following we will build a mathematical model based on the paradigm of a multifractal theory of motion for the analysis of a complex polymer-drug dynamics. Therefore, admitting that from both structural and functional perspectives the polymer-drug complex system is assimilated to a multifractal system [26-29] the wide ranges of drug release dynamics can be described through the movement of the so-called polymer-drug complex system entities (or structural units) on trajectories that contain nondifferentiability (multifractal curves). Accepting multifractality as a fundamental property in drug release dynamics (and since multifractality is induces through stochasticity [30, 31]), the drug release dynamics can be associated to various flow regimes of a stochastic fluid at various scale resolutions. For a large temporal scale resolution, with respect to the inverse of the highest Lyapunov exponent, the deterministic trajectories of the polymer-drug system structural units can be replaced by a collection of potential trajectories (virtual trajectories), while the concept of definite trajectories can be replaced by that of probability density. In such a context a multifractal probability density conservation law will become functional [30-32] for the drug release dynamics. If we assume that multifractalizaion is 
achieved through stochasticization, then the one-dimensional density probability multifractal law takes the form:

with

$$
\partial_{t} P+\partial_{x} j_{x}=0
$$

$$
\partial_{t}=\frac{\partial}{\partial t}, \partial_{x}=\frac{\partial}{\partial x}
$$

In the above relations $P(x, t, d t)$ is the probability density, $j_{x}(x, t, d t)$ is the probability current density, $x(t, d t)$ is the spatial coordinate, $t$ is the temporal coordinate and $d t$ is the scale resolution. From a mathematical perspective $P, j_{x}$ and $x$ are multifractal variables, while $t$ is a non-multifractal variable having the affine parameter role for the movement curves $[23,25,26]$. The probability current density must contain both the drift multifractal component:

$$
j_{x}^{d r i f t}(x, t, d t)=\mu(x, t, d t) P(x, t, d t)
$$

and the Fickian multifractal component (the diffusion one):

$$
j_{x}^{\text {diffusion }}(x, t, d t)=-\frac{1}{2} \partial_{x}\left[\sigma^{2}(x, t, d t) P(x, t, d t)\right]
$$

where $\mu(x, t, d t)$ and $\sigma^{2}(x, t, d t)$ are multifractal variables. The probability density dynamics are determined only through $\mu$ and $\sigma^{2}$; this results from the explicit form of (1), taking into account relations (2) and (3), i.e:

$$
\partial_{t} P(x, t, d t)+\partial_{x}\left\{\mu(x, t, d t) P(x, t, d t)--\frac{1}{2} \partial_{x}\left[\sigma^{2}(x, d t) P(x, d t)\right]\right\}
$$

Equation (5) can be analytically solved only for particular cases. By choosing:

$$
\begin{gathered}
\mu=-\eta x, \eta=\bar{\eta}(d t)^{\left[\frac{2}{f(\alpha)}\right]-1} \\
\sigma^{2}=-\lambda, \lambda=\bar{\lambda}(d t)^{\left[\frac{2}{f(\alpha)}\right]-1}
\end{gathered}
$$

and through integration we obtain the following solution:

$$
P(x, t, d t)=\frac{1}{\left\{2 \pi \frac{\lambda}{\eta}[1-\exp (-2 \eta t)]\right\}^{1 / 2}} \exp \left\{-\frac{\left[x-x_{0} \exp (-\eta t)\right]^{2}}{2 \frac{\lambda}{\eta}[1-\exp (-2 \eta t)]}\right\}
$$

In relations (6) (7) and (8) $f(\alpha)$ is the singularity spectrum of order $\alpha$ and $\alpha$ is the singularity index through which the fractal dimension $D_{F}$ is specified (for $D_{F}$ we can use any definitions - Kolmogorov fractal dimension, Hausdorff-Besikovich fractal dimension etc. [34]; it is regularly found that $D_{F}<2$ for correlative processes and $D_{F}>2$ for non-correlative processes). From such a perspective, through $f(\alpha)$ it is possible to identify not only the areas of drug release that are characterized by a certain fractal dimension (i.e. the case of mono-fractal drug release dynamics), but also the number of areas for which the fractal dimensions are situated in an interval of values (i.e. the case of multifractal drug release dynamics). More than that, though the same $f(\alpha)$, it is possible to identify classes of universality in the drug release dynamics laws, even when regular or strange attractors have various aspects [30, 31].

Therefore, equation (8) is a multifractal Gaussian (dependent on the scale resolution) for which the central values, still dependent on scale resolution, decrease exponentially towards zero $(\eta>0)$ and its variation asymptotically goes through $(\lambda / \eta)$. In particular, in the case of mono-fractal drug release 
dynamics, described through Peano type curves, i.e. $f(\alpha) \rightarrow 2$, the solution (8) can be reduced to the standard form $[30,31]$ :

$$
P(x, t)=\frac{1}{\left\{2 \pi \frac{\bar{\lambda}}{\overline{\bar{\eta}}}[1-\exp (-2 \bar{\eta} t)]\right\}^{1 / 2}} \exp \left\{-\frac{\left[x-x_{0} \exp (-\bar{\eta} t)\right]^{2}}{2 \frac{\bar{\lambda}}{\overline{\bar{\eta}}}[1-\exp (-2 \bar{\eta} t)]}\right\}
$$

Relation (8) can be written in a more simplified way if we introduce the non-dimensional variable:

and the non-dimensional parameter

$$
\xi=\frac{x}{x_{0}}, \tau=\eta t
$$

It results

$$
v=\frac{x}{\eta x_{0}}
$$

$$
\rho(\xi, \tau, v)=\frac{1}{\{v[1-\exp (-2 \tau)]\}^{1 / 2}} \exp \left\{-\frac{\left[\xi-\xi_{0} \exp (-\tau)\right]^{2}}{2 v[1-\exp (-2 \tau)]}\right\}
$$

From here, considering the physical significance of $\rho[30,32]$, by multiplying (12) with a nondimensional constant playing the role of rest mass of the complex polymer-drug systems entities, we can write the multifractal law that can generate the release dynamics as:

$$
\frac{M(\tau)}{M_{\infty}}=A \rho(\xi, \tau, v), A=\text { const }
$$

\section{Results and discussions}

A series of four amphiphilic formulations with different mass ratios of the hydrophilic/hydrophobic structural blocks and the same amount of model drug were prepared by condensation reaction of amines with aldehydes, and their drug release behavior was tested into an in vitro physiologic environment [9, 33].

By spectroscopic and microscopic measurements it was established that the drug has been finely dispersed into the chitosan based matrix due to the strong electrostatic and H-bond forces which hampered the natural tendency of crystallization of the drug. This can be easily seen in the polarizing microscopy (POM) images in Figure 1 which showed a continuous fine birefringent texture and no obvious drug crystals, which clearly indicate no phase separation of the drug in the matrix [34].
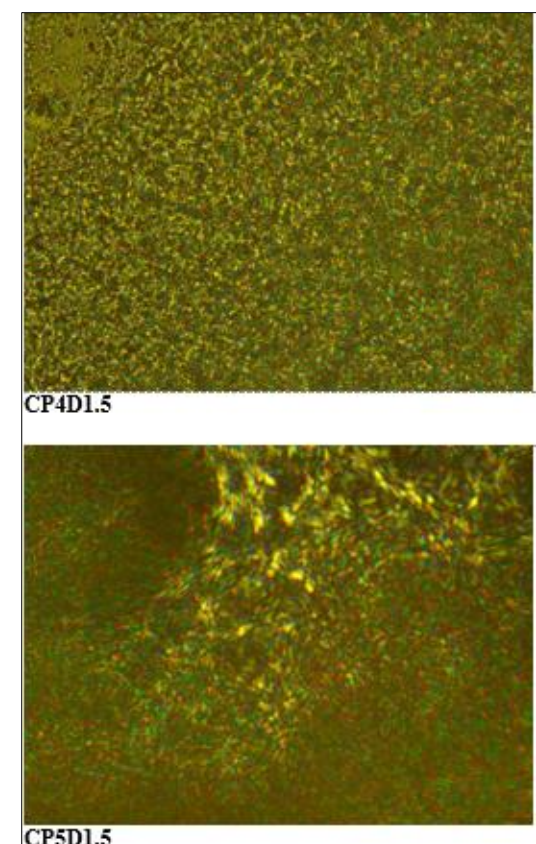

CP5D1.5
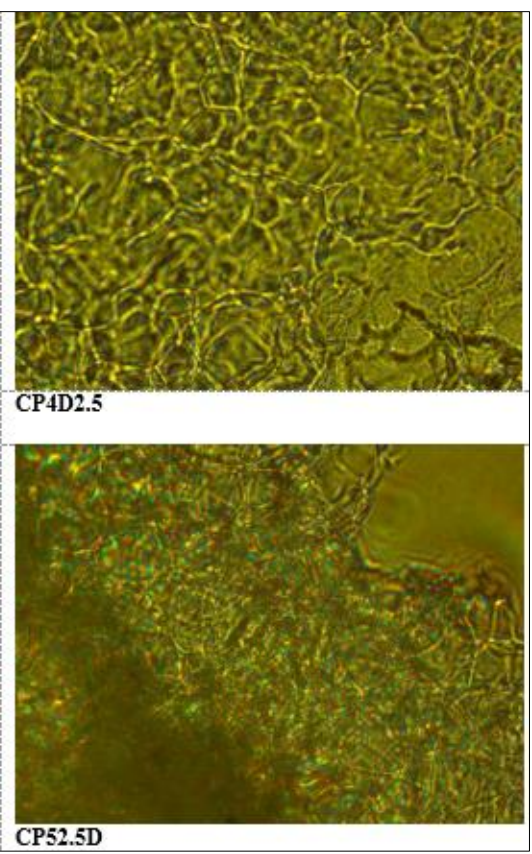

Figure 1. POM images of the amphiphilic formulations 
The formulations displayed a porous morphology, with interconnected pores which assure favorable sink conditions of the drug (Figure 2). Moreover, the scanning electron microscopy (SEM) images confirmed the POM observations, indicating no sub-micrometric crystals into the pores or on the pore walls, suggesting a good dispersion of the drug into the matrix $[35,36]$.
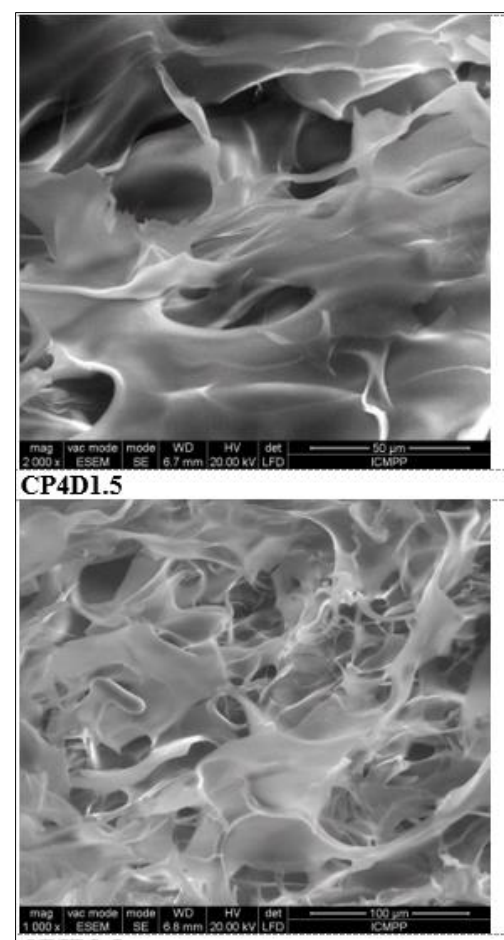

CP5D1.5

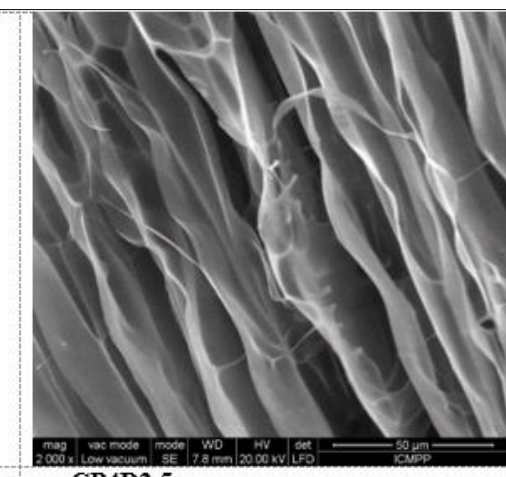

CP4D2.5

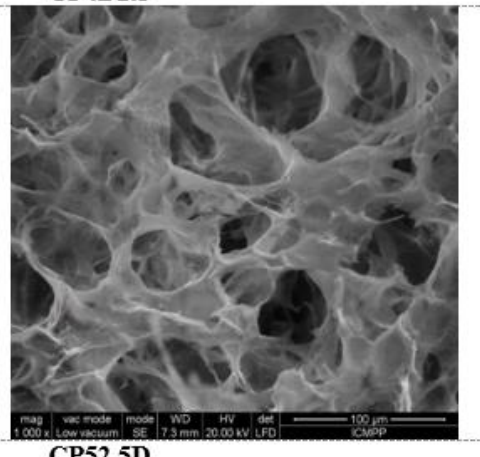

CP52.5D

Figure 2. SEM images of the drug release formulations

The in vitro investigation of the drug release indicated the influence of the hydrophobic/hydrophilic balance on the release rate (Figure 3). It can be observed that the progressive increase of the hydrophilic component into formulation lead to the fastening of the drug release. Thus, the simple manipulation of the hydrophobic/hydrophilic balance can tune the drug release in agreement with the addressed requirement [9, 35-37]. Quantitatively speaking, in 7 days the CP4B2.5D sample released more than 99\% from the entire amount of the encapsulated DCF, while the sample CP5B1.5D released only 91\% (Figure 3).

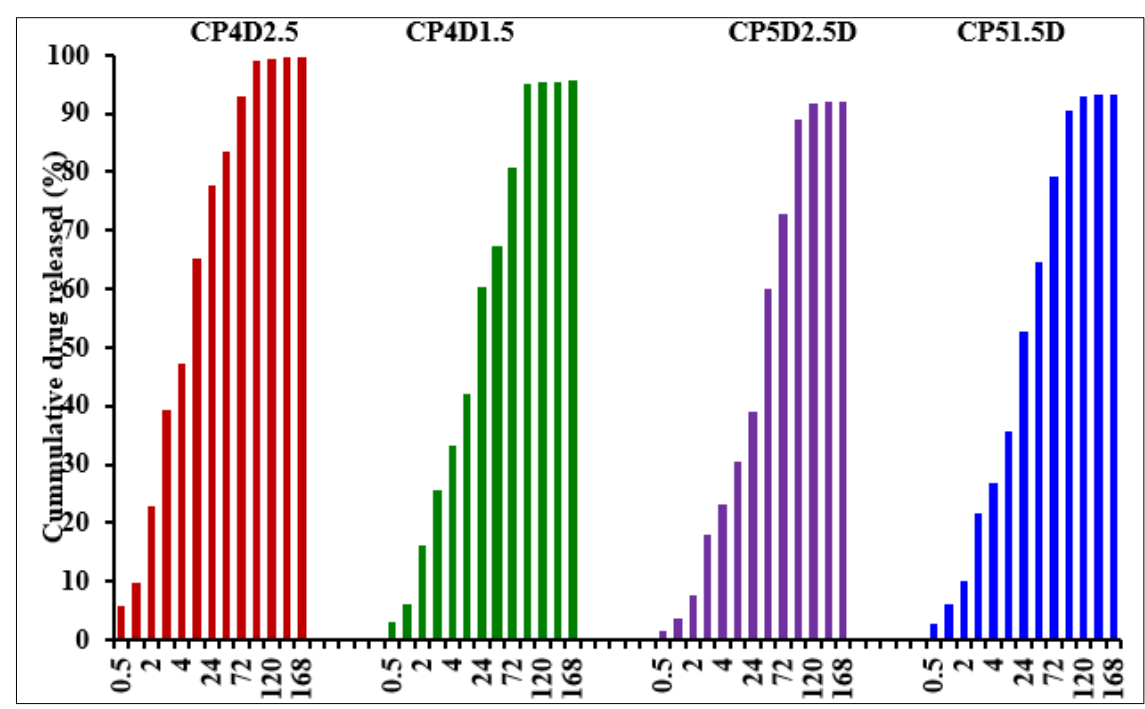

Figure 3. The in vitro drug release in a medium mimicking the physiologic medium 
The fitting of the in vitro release data on the five traditional mathematical equations didn't conclude on the mechanism of the drug release, as all the equations fitted very well, indicating that many factors are influencing the delivery process. Concerning the theoretic model developed in section 3, this can be validated through an adequate calibration of the empirical data, by choosing the constants according to the particularities of our polymer-drug system followed by a normalization of the data. The calibration process is not a trivial one as it strictly depends on the nature of the investigated phenomena. This method was previously tested for other physical phenomena with promising results [38-40]. We can observe that the model fits well the CPD2.5, where the saturation region is reached earlier. This is also due to the morphology of the formulation which has a more organized structure enhancing the release. This, translated into the fractal paradigm used for the theoretical model, means that a non-fractal morphology will lead to a higher fractality in the geodesics of the release drugs as it enhances the interactions between the drug and the release media. As the morphology of the polymer formulations becomes fractalized the release is reduced and the overall fractalization degree of the drug-release is reduced (Figure 4).

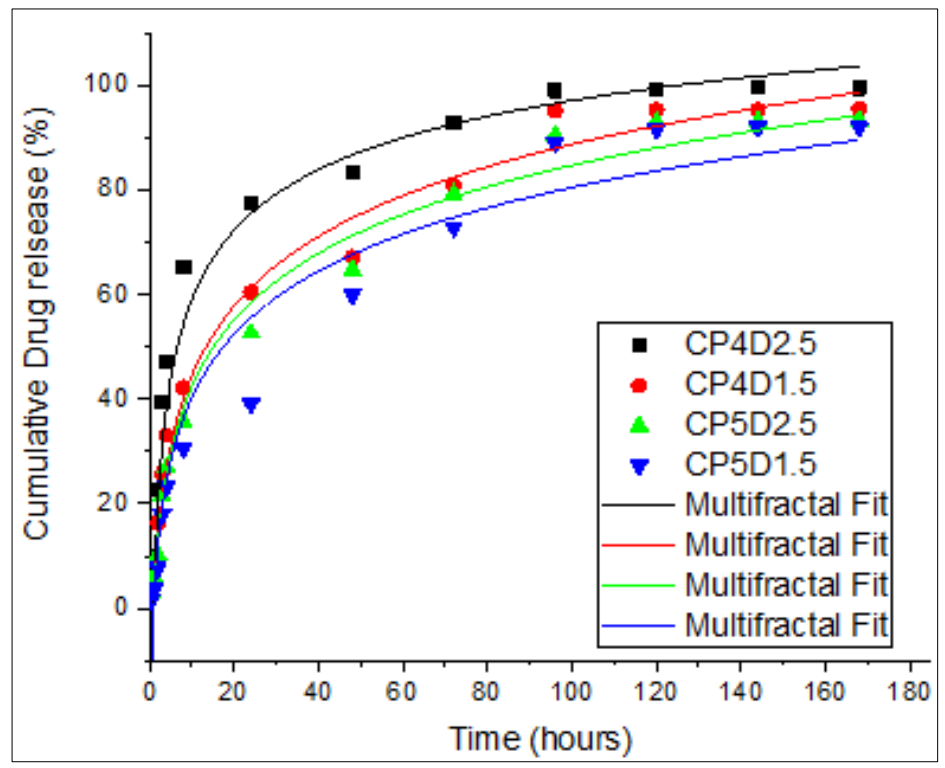

Figure 4. Multifractal theoretical fir of drug release in physiological like medium

Other authors reached similar results with respect to the presented theoretical model of drug delivery systems and controlled drug release by using similar mathematical procedures, but further studies are needed in order to increase the application of this methods [41-46].

In the following we would like to present about the perspectives of NSAIDSs controlled release in the field of gynecology. NSAIDs (such as diclofenac) are widely employed first choice drugs for treatment of different medical afflictions in the field obstetrics and gynecology domain. The traditional NSAIDs action mechanism is the inhibition of the prostaglandin $\mathrm{G} / \mathrm{H}$ synthase enzymes, also known as the cyclooxygenases. These enzymes convert arachidonic acid to the unstable intermediates prostaglandin $\mathrm{G} 2$ and prostaglandin $\mathrm{H} 2$, leading to the production of thromboxane A2 and a variety of other prostaglandins which contribute to pain. In higher concentrations, NSAIDs are also known to reduce the production of superoxide radicals, induce apoptosis, inhibit the expression of adhesion molecules, decrease nitric oxide synthase, decrease proinflammatory cytokines, modify lymphocyte activity and alter cellular membrane functions. In obstetrics and gynecology, NSAID's have long been used to control acute and chronic postoperative pain, menstrual pain, pain related to medical abortions, menorrhagia, intrauterine device, assist in fertility treatment, and administered as tocolytics in preterm labor [47]. 
When using nonsteroidal anti-inflammatory drugs (NSAIDs) for analgesic purposes, drugs represent the main treatment choice in gynecology inflammatory diseases and acute or chronic pelvic pain. Endometriosis or intrauterine adhesions are two frequent inflammatory diseases in which the common symptom is represented by cyclic pelvic pain that often requires the use of NSAIDs. Pharmacotherapy plays an important role in the management of these two pathologies with long-term treatment administration compared with clinical efficacy as pain control and recurrence prevention after surgical treatment [48]. Non-steroidal anti-inflammatory drugs poses anti-inflammatory, antipyretic and analgesic features. The action of NSAIDs is to inhibit the cyclooxygenase (COX), an enzyme that have two isoforms - COX-1 and COX-2, which are responsible for creating prostaglandins. Under physiological circumstances, the expression of COX-1 forms prostaglandins whilst COX-2 is expressed following pathophysiological conditions in injured tissues in order to form prostaglandins. The mechanism of action is to block these isoforms but their selectivity varies between. However, the efficacy and related side effects are mostly due to their common action pathways [49].

Drug delivery systems are becoming more achievable in the gynecological field due to their capacity to decrease various side effects of the drugs. In our opinion, after further studies, our model could be employed for developing new controlled drug release mechanism for the therapeutic use of NSAIDs in gynecology.

\section{Conclusions}

An amphiphilic matrix based on chitosan was developed and its ability for controlled drug release applications was investigated. The investigated systems demonstrated a lack of any in vivo toxicity encouraging further investigations of the laws which governs their ability to function as an efficient matrix. However future studies are need it in order to establish its applications in endometriosis or intrauterine adhesions management. A theoretical model was built on a set of multifractal conservations laws for density probability considering a manifestation of the multifractality through stochasticity. In this complex paradigm the multifractal-continuous functions correspondence is ruled by the release modes.

\section{References}

1.JOMMANEE N., CHANTHA C., MANOKRUANG K., Preparation of injectable hydrogels from temperature and $\mathrm{pH}$ responsive grafted chitosan with tuned gelation temperature suitable for tumor acidic environment. Carbohydr Polym, 2018, 198: 486-494.

2.CHEN S., WU M., WANG C., YAN S., LU P., WANG S., Developed Chitosan/Oregano Essential Oil Biocomposite Packaging Film Enhanced by Cellulose Nanofibril, Polymers, 2020, 12(8): 1780.

3.CHEN A., SAMANKUMARA L.P., GARCIA C., BASHAWA K., WANG G., Synthesis and characterization of 3-O-esters of $\mathrm{N}$-acetyl-d-glucosamine derivatives as organogelators, New Journal of Chemistry, 2019, N 43: 7950-7961.

4.KUEN C.Y., GALEN T., FAKURAZI S., OTHMAN S.S., MASARUDIN M.J., Increased Cytotoxic Efficacy of Protocatechuic Acid in A549 Human Lung Cancer Delivered via Hydrophobically ModifiedChitosan Nanoparticles as an Anticancer Modality, Polymers, 2020, 12(9): 1951.

5.ZAKARIA L., WONG T.W., ANUAR N.K., et al., Enhancing sustained drug release property of chitosan in spheroids through crosslinking reaction and coacervation, Powder Technology, 2019, 354: 815-821.

6.OLARU A.-M., MARIN L., MORARIU S., et al., Biocompatible chitosan based hydrogels for potential application in local tumour therapy, Carbohydrate Polymers, 2018, 179: 59-70.

7.AILINCAI D., TARTAU MITITELU L., MARIN L., Drug delivery systems based on biocompatible imino-chitosan hydrogels for local anticancer therapy, Drug Deliv, 2018, 25: 1080-1090.

8.CRACIUN A.M., MITITELU TARTAU L., PINTEALA M., MARIN L., Nitrosalicyl-imine-chitosan hydrogels based drug delivery systems for long term sustained release in local therapy, J Colloid Interface Sci, 2019, 536:196-207. 
9.AILINCAI D., MITITELU-TARTAU L., MARIN L., Citryl-imine-PEG-ylated chitosan hydrogels Promising materials for drug delivery applications, International Journal of Biological Macromolecules, 2020, 162: 1323-1337.

10.IFTIME M.-M., MITITELU TARTAU L., MARIN L., New formulations based on salicyl-iminechitosan hydrogels for prolonged drug release, International Journal of Biological Macromolecules, 2020, 160: 398-408.

11.WU Y., ANIA RASHIDPOUR A., ALMAJANO M.P., METÓN I., Chitosan-Based Drug Delivery System: Applications in Fish Biotechnology, Polymers, 2020, 12(5): 1177.

12.GUYOTA C., CERRUTI M., LEROUGE S., Injectable, strong and bioadhesive catechol-chitosan hydrogels physically crosslinked using sodium bicarbonate, Materials Science and Engineering, 2021, C 118: 111529.

13.CHAIWONG N., LEELAPORNPISID P., JANTANASAKULWONG K., et al., Antioxidant and Moisturizing Properties of Carboxymethyl Chitosan with Different Molecular Weights, Polymers, 2020, 12(7): 1445.

14.BRAUN J., BARALIAKOS X., WESTHOFF T., Non-steroidal anti-inflammatory drugs and cardiovascular risk - a matter of indication. Semin Arthritis Rheum, 2020, 50(2): 285-288.

15.BHALA N., EMBERSON J., MERHI A., et al.,_Vascular and upper gastrointestinal effects of nonsteroidal anti-inflammatory drugs: meta-analyses of individual participant data from randomised trials, Lancet, 2013, 382(9894): 769-779.

16.SCHMIDT M., SORENSEN H.T. AND PEDERSEN L., Diclofenac use and cardiovascular risks: series of nationwide cohort studies, BMJ, 2018, 362:k3426.

17.AILINCAI D., GAVRIL G., MARIN L., Polyvinyl alcohol boric acid - A promising tool for the development of sustained release drug delivery systems, Materials Science and Engineering: C, 2020,107: 110316.

18.TIWARI G., TIWARI R., SRIWASTAWA B., BHATIL., PANDEY S., PANDEY P., BANNERJEE S.K., Drug delivery systems: An updated Review, Int. J. Pharm. Investig., 2012, 2(1): 2-11.

19.PATRA J.K., DAS G., FRACETO L.F, CAMPOES E.V.R., TORRES M.P.R, ACOSTA-TORRES L.S., Nano based drug delivery systems: recent developments and future perspectives, J. Nanobiotechnology, 2018, 16: 17.

20.FISHER O.Z., KHADEMHOSSEINI A., PEPPAS N.A., Drug delivery: nanoscale devices, in Encyclopedia of Materials: Science and Technology, 2010, 1: 9.

21.PEPPAS N.A., BRANNON-PEPPAS L., Drug delivery Biomaterials in Encyclopedia of Materials: Science and Technology (Second Edition), 2001, 2: 196-207.

22.KOSMIDIS K., ARDYRAKIS P., MACHERAS, P., Fractal kinetics in drug release from finite matrices, The journal of Chemical Physics, 2003, 119(2): 6373-6377.

23.AGOP, M., MERCHES I., Operational procedures describing physical systesm, CRC press, Taylor and Francis Group, 2019.

24.NOTTALE L., Scale Relativity and Fractal Space-Time: A New Approach to Unifying Relativity and Quantum Mechanics, Imperial College Press, London, 2011.

25.M. HONCIUC, V.P. PAUN, Liquid crystal-like behavior of some fatty acids mixtures, Rev. Chim., 54(1), 2003, 74-76.

26.LAZAR B., STERIAN A., PUSCA S., et al., Simulating delayed pulses in organic materials, Conference: International Conference on Computational Science and Its Applications (ICCSA 2006), Computational Science and Its Applications - ICCSA 2006, PT 1 Book Series: LECTURE NOTES IN COMPUTER SCIENCE, 2006, 3980: 779-784.

27.IFTIME M. M., DOBRECI D. L., IRIMICIUC S. A., AGOP M., PETRESCU T., DOROFTEI B., A theoretical mathematical model for assessing diclofenac release from chitosan-based formulations, Drug Delivery, 2020, 27(1): 1125-1133. 
28. AILINCAI D., DOROBANTU AM., DIMA B., IRIMICIUC SA., LUPASCU C., AGOP, M., OLGUTA O., Poly(vinyl alcohol boric acid)-Diclofenac Sodium Salt Drug Delivery Systems: Experimental and Theoretical Studies, Journal of Immunology Research, 2020, 3124304.

29.IANCU R., IRIMICIUC S.A., AGOP M., FRASILA M., PAUN M.A., PAUN V.A., V.P. PAUN, STRATULAT S., 5-Fluorouracil Release from Chitosan-Based Matrix. Experimental and Theoretical Aspects, Mater. Plast., 57(3), 2020, 180-189.

30.JACKSON, E. A., Perspectives of Nonlinear Dynamics, Vol. 1, Cambridge University Press, New York, 1993.

31.NICHITA, M.V., PAUN, M.A., PAUN, V.A., PAUN, V.P., Fractal Analysis of Brain Glial Cells. Fractal Dimension and Lacunarity, University Politehnica of Bucharest Scientific Bulletin-Series AApplied Mathematics and Physics, 2019, 81(1): 273-284.

32.MANDELBROT, B. B., The Fractal Geometry of Nature, W. H. Freeman and Co., San Fracisco, 1982.

33.MARIN L., AILINCAI D., MARES M., et al., Imino-chitosan biopolymeric films. Obtaining, selfassembling, surface and antimicrobial properties, Carbohydrate Polymers, 2015, 117: 762-770.

34.BORDESCU D., PAUN M.A., PAUN V.A., PAUN V.P., Fractal analysis of Neuroimagistic. Lacunarity degree, a precious indicator in the detection of Alzheimer's disease, University Politehnica of Bucharest Scientific Bulletin, Series A-Applied Mathematics and Physics, 2018, 80(4): 309-320.

35.YE J., FU S., ZHOU S., et al., Advances in hydrogels based on dynamic covalent bonding and prospects for its biomedical application, European Polymer Journal, 2020, 139: 110024.

36.AHSAN A., FAROOQ M.A., PARVEEN A., Thermosensitive Chitosan-Based Injectable Hydrogel as an Efficient Anticancer Drug Carrier, ACS Omega 2020, 5: 20450-20460.

37.LALL, A., TAMO K., DOENCH I., et al., Nanoparticles and Colloidal Hydrogels of ChitosanCaseinate Polyelectrolyte Complexes for Drug-Controlled Release Applications, Int. J. Mol. Sci., 2020, 21(16): 5602.

38.IRIMICIUC S.A., NICA P.E., AGOP M., FOCSA, C. Target properties - Plasma dynamics relationship in laser ablation of metals: Common trends for fs, ps and ns irradiation regimes Appl. Surf. Sci., 2020, 506: 144926.

39.IRIMICIUC S., BULAI G., AGOP M., GURLUI S., Influence of laser-produced plasma parameters on the deposition process: In situ space- and time-resolved optical emission spectroscopy and fractal modeling approach, Appl. Phys. A Mater., 2018, 124: 615.

40.COBZEANU B.M., IRIMICIUC S., VAIDEANU D., GRIGOROVICI A., POPA O., Possible Dynamics of Polymer Chains by Means of a Ricatti's Procedure-an Exploitation for Drug Release at Large Time Intervals, Mater. Plast., 54(3), 2017, 531-534.

41.AGOP, M., MURGULET, C., El Naschie's epsilon((infinity)) space-time and scale relativity theory in the topological dimension $\mathrm{D}=4$, CHAOS SOLITONS \& FRACTALS Volume: 32 Issue: 3 Pages: 1231-1240 Published: MAY 2007

42.PAUN M.A., HANU R.C., NICANOR C. et al, Internal Friction Phenomena at Polymeric and Metallic Shape Memory Materials. Experimental and Theoretical Results, Mater. Plast., 47(2), 2010, 209-214.

43.AGOP M., IOANNOU P., NICA P. et al., Fractal characteristics of the solidification process, Materials transaction. Materials Transactions, 2004, 45(3): 972-975.

44.BUJOREANU C., NEDEFF F., BENCHEA M., et al. Experimental and theoretical considerations on sound absorption performance of waste materials including the effect of backing plates, Applied Acoustics, 2017, 119: 88-93.

45.AGOP M., PAUN V., HARABAGIU A., El Naschie`s ((infinity)) theory and effects of nanoparticle clustering on the heat transport in nanofluids. Chaos solitons \& fractals, 2008, 37(5): 1269-1278.

46.COLOTIN M., POMPILIAN G. O., NICA P. et al., Fractal Transport Phenomena through the Scale Relativity Model, Acta Physica Polonica A, 2009, 116(2): 157-164. 
47.LIVSHITS A., SEIDMAN D. S., Role of Non-Steroidal Anti-Inflammatory Drugs in Gynecology, Pharmaceuticals (Basel), 2010, 3(7): 2082-2089.

48.CIBOTARU S., SANDU A.-I., BELEI D., MARIN L., Water soluble PEGylated phenothiazines as valuable building blocks for bio-materials, Materials Science and Engineering: C, 2020, 116: 111216. 49.BEDAIWY M.A., ALLAIRE C., YONG P. AND ALFARAJ S., Medical management of endometriosis in patients with chronic pelvic pain, Semin Reprod Med, 2016, 35(1): 38-53.

$\overline{\text { Manuscript received: } 22.10 .2020}$ 